\title{
GESTIÓN DEL CONOCIMIENTO EN LA CARRERA DE EDUCACIÓN PRIMARIA EN LA UNIVERSIDAD NACIONAL DEL SANTA, PERÚ
}

\author{
Romy Mas* \\ https://orcid.org/0000-0001-9244-6656 \\ Ruth Meregildo** \\ https://orcid.org/0000-0001-6706-4752 \\ Charles Torres*** \\ https://orcid.org/0000-0002-5716-2575 \\ Reemberto Cruz $* * * *$ \\ https://orcid.org/0000-0003-2362-2147
}

RECIBIDO: Septiembre 2020 / ACEPTADO: Diciembre 2020 / PUBLICADO: Mayo 2021

Como citar: Mas, Romy; Meregildo, Magna; Torres, Charles y Cruz, Reemberto. (2021). Gestión del conocimiento en la carrera de educación primaria en la Universidad Nacional del Santa, Perú. Telos: revista de Estudios Interdisciplinarios en Ciencias Sociales, 23 (2), Venezuela. (Pp.207-226).

DOI: www.doi.org/10.36390/telos232.02

\section{RESUMEN}

La gestión del conocimiento es un proceso ejecutado en diversidad de empresas y organizaciones; posee tres dimensiones importantes: creación; transferencia almacenamiento; aplicación y uso del conocimiento. Autores como: Escorcia y Barros (2020); Bom y Bolívar (2015); Tarí y García (2009), concuerdan en la necesidad de aumentar la calidad y valoración del capital intelectual de las empresas con miras a aumentar la generación de conocimiento y por ende mejorar la gestión. El objetivo del estudio fue proponer una herramienta para mejorar la gestión del conocimiento en la carrera de educación primaria de la Universidad Nacional del Santa, Perú. La investigación fue descriptiva con diseño de campo; la población fue de 120 estudiantes de la carrera de educación primaria y se les aplicó un cuestionario validado por expertos de confiabilidad: 0.896/bueno. Como resultado se obtuvo que el $60.8 \%$ de los estudiantes, percibe la gestión como regular. Se concluye con esta investigación que se debe mejorar la gestión del conocimiento, ameritando cambios en los procesos y procedimientos, por medio de la implementación de la herramienta de mejora continúa propuesta en esta investigación.

\footnotetext{
* Docente - Investigadora, Universidad Nacional del Santa, Facultad de Educación y Humanidades. Correo Electrónico rkmas0220@gmail.com

* Docente - Investigadora, Universidad Nacional de Trujillo, Facultad de Educación, Departamento de Ciencias de la Educación. Correo Electrónico: rmeregildo@unitru.edu.pe

*** Docente - Investigador, Universidad Nacional Mayor de San Marcos, Escuela de Post Grado. Correo Electrónico: charlestorres15@yahoo.com

**** Docente - Investigador, Universidad Católica de Trujillo "Benedicto XVl", Facultad de Educación y Humanidades. Correo Electrónico: r.cruz@uct.edu.pe
} 
Gestión del conocimiento en la carrera de educación primaria en la Universidad Nacional del Santa, Perú

Palabras Clave: Gestión del conocimiento, carrera de educación primaria, mejora continua, ciclo de Deming.

\title{
Knowledge management model in the primary Education career
}

\begin{abstract}
Knowledge management is a process carried out in a diversity of companies and organizations; it has three important dimensions: creation; transfer - storage; application and use of knowledge. Authors such as: Escorcia and Barros (2020); Bom and Bolívar (2015); Tarí and Garcia (2009), agree on the need to increase the quality and valuation of the intellectual capital of companies in order to increase the generation of knowledge and therefore improve management. The objective of the study was to propose a tool to improve knowledge management in the primary education career of the National University of Santa, Peru. The research was descriptive with a field design; the population consisted of 120 primary school students and a questionnaire validated by reliability experts was applied: 0.896 / good. As a result, it was obtained that $60.8 \%$ of the students perceive the management as regular. This research concludes that knowledge management should be improved, meriting changes in processes and procedures, through the implementation of the continuous improvement tool proposed in this research.
\end{abstract}

Key Words: Knowledge management, primary education career, continuous improvement, Deming cycle

\section{Introducción}

El logro de un fin supremo como lo es el educarse, es un hecho con el cual ha de identificarse y al que debe aspirar toda sociedad moderna; en cuanto a esto, la UNESCO ha establecido como paradigma educativo el siguiente: Educación para todos, durante toda la vida. El mismo es algo difícil de lograr, al decir de algunos autores, cuando se ignora y no se reconoce el rol de la gestión educativa estratégica, en el sentido de la integración de la gestión del conocimiento, la ciencia, la tecnología y la innovación en la educación superior (Barbón y Fernández, 2017).

En base a lo anterior, en la actualidad existe un consenso mundial sobre la importancia del desarrollo del conocimiento, como eje del crecimiento de las organizaciones, de la economía y por ende de los países. Por tal motivo, en los últimos tiempos se ha dado prioridad a la creación de conocimiento para impulsar y desarrollar las sociedades, planteándose un sin número de mecanismos para llevar a cabo su gestión, como lo son en esencia, la creación, almacenamiento, difusión, aplicación y el uso del conocimiento.

En el caso de las Instituciones de Educación Superior (IES), la creación y transmisión del conocimiento se orienta más hacia la ciencia y la universalidad por medio de la investigación científica, por tal razón, su misión de crear y difundir conocimiento está dominada por el entendimiento explícito (Escorcia y Barros, 2020).

En el ámbito de estudio de la gestión del conocimiento, existen trabajos que han servido de referencia como por ejemplo, los anteriores autores Escorcia y Barros, (2020), quienes 
infieren en su estudio, que con la finalidad de responder a los cambios globales, las universidades deben contribuir a elevar la calidad del capital intelectual, en busca del fomento y la creación de nuevos conocimientos, que a su vez se convertirán en la fuerza de trabajo para la innovación.

También en su trabajo, Pérez, (2016), indica que la gestión del conocimiento, es la disciplina encargada de diseñar e implementar modelos de gestión que permiten identificar, capturar y compartir el conocimiento entre los miembros de una organización, impulsando la creación de valor y generación de ventajas competitivas. Al mismo tiempo una gestión del conocimiento exitosa lleva a las organizaciones a la mejora continua, aspecto este vital para alcanzar los objetivos planteados. Siendo relevante indagar e identificar un modelo; como instrumento, aporte o propuesta, para la mejora de la gestión (Briceño, Correa, Valdés y Hadweh, 2020).

Por lo antes expresado, cobra importancia la realización de ésta investigación; en la cual se aborda la situación planteada por los estudiantes al plantear sus percepciones en cuanto a la mejora de la gestión del conocimiento, en la carrera de educación primaria de la Universidad Nacional del Santa (UNS), Perú. Esta universidad brinda una formación académica y educativa, con la mayor concentración de esfuerzo en el logro de competencias y dominio de los saberes; integrando actitudes y capacidades; además de que la Escuela Profesional de Educación Primaria (EEP), forma docentes investigadores capaces de conducir el proceso de enseñanza-aprendizaje, con niveles óptimos de calidad, eficiencia, eficacia y pertinencia (Universidad Nacional del Santa, 2020).

En ese sentido, es prioritario atender al llamamiento que realizan los estudiantes de dicha escuela, quienes expresan la necesidad de mejorar los procesos de la gestión del conocimiento, en vista de que prevalecen deficiencias desde el punto de vista de la gestión propiamente dicha. Por lo que es pertinente la aplicación de métodos y procedimientos idóneos, para llevar a cabo una gestión del conocimiento enmarcada en la implementación de una herramienta para la mejora continua, que incluya a todos los miembros de la institución implicados en estos procesos.

La descripción de la problemática existente, lleva a formular la siguiente interrogante: ¿Qué herramienta se puede implementar para mejorar la Gestión del Conocimiento de la carrera de Educación Primaria de la Universidad Nacional del Santa, en Perú?. Planteándose como objetivo general del estudio: Proponer una herramienta para mejorar la gestión del conocimiento en la Carrera de Educación Primaria de la Universidad Nacional del Santa, en Perú.

\section{Gestión del Conocimiento}

A medida que las organizaciones interactúan con sus entornos, absorben información, la convierten en conocimiento y llevan a cabo acciones sobre la base de la combinación de ese conocimiento y de sus experiencias, valores y normas internos; sienten y responden. Sin conocimiento, una organización no se podría organizar a sí misma (Fernández y Soto, 2015).

Entonces, teniendo claro que el conocimiento es, el activo intangible más valioso en toda institución; de cualquier naturaleza, pública o privada; su manejo implica la activación de nuevas formas de trabajar, capacitar, educar, aplicar nuevos métodos y enfoques de modo que la interacción entre las personas sea más efectiva y se obtengan mejores resultados; los 
Gestión del conocimiento en la carrera de educación primaria en la Universidad Nacional del Santa, Perú

cuales redunden en mayor productividad. De este hecho, deben ser conscientes hoy día todos los interesados en el desarrollo organizacional y por ende de las sociedades, más aún en el ámbito de la educación superior.

Ahora bien, la gestión del conocimiento es una disciplina del campo de la administración, que se ha venido ampliando en su ejecución, hasta llegar a desarrollarse formalmente incluso en las instituciones de educación superior. Tomando en cuenta que es en las IES donde se origina y desarrolla el "conocimiento" propiamente dicho, se ha adoptado la terminología tal como se le asigna en el ámbito empresarial, y de alguna manera la connotación se asimila en la realidad de las universidades, con el afán de alinear procesos académicos e investigativos, con el desarrollo, la innovación y la globalización.

En cuanto a su significado, la gestión del conocimiento tiene varias acepciones, válidas todas y pertinentes, a la hora de aplicar el proceso de gestionar en diversas situaciones y circunstancias organizacionales. Por lo antes dicho se mencionan las definiciones descritas por algunos autores (Cuadro 1) que han tratado la temática en diversos estudios.

\section{Cuadro 1. Definiciones sobre Gestión del Conocimiento}

\begin{tabular}{|c|c|}
\hline \multicolumn{2}{|r|}{ Gestión del Conocimiento } \\
\hline $\begin{array}{l}\text { Escercla y Barres: } \\
\text { (2020) }\end{array}$ & 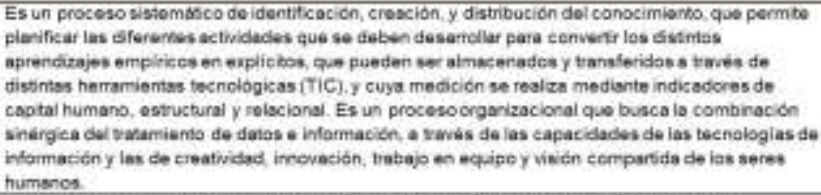 \\
\hline $\begin{array}{l}\text { Akbon, Y Farnindar } \\
\text { a2017 }\end{array}$ & 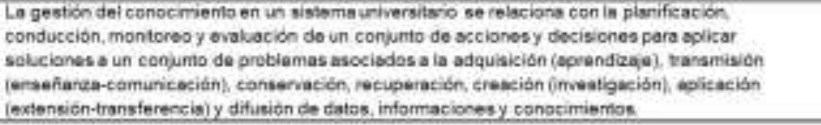 \\
\hline $\begin{array}{l}\text { lase: } \\
\text { Herremans y } \\
\text { Nazarl }(2+0) \text {. }\end{array}$ & 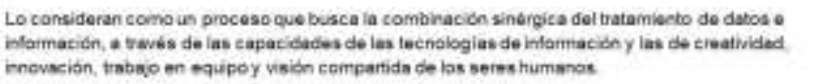 \\
\hline $\begin{array}{l}\text { Godoy, Naitha, Wora, } \\
\text { Jeneth, Y Uberlo, } \\
\text { Francisco, t2017) }\end{array}$ & 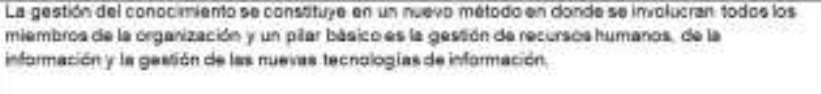 \\
\hline $\begin{array}{l}\text { Mega, Veava y Gaviris } \\
\text { (2018) }\end{array}$ & 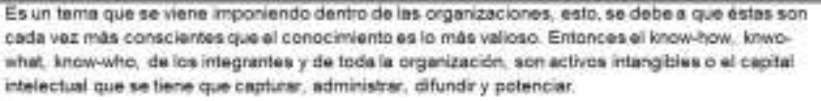 \\
\hline
\end{tabular}

Fuente: Elaboración propia, basado en: Escorcia y Barros (2020); Barbón y Fernández (2017); Isaac, Herremans y Nazari (2017), Godoy, Mora y Liberio (2017); Mejía, Vesga y Gaviria (2018).

Tomando como referencia las definiciones anteriores, la gestión del conocimiento en las Instituciones de Educación Superior (IES), constituye un proceso de orientación y promoción de la actividad científica y la producción de conocimiento. De acuerdo a esto la gestión del conocimiento que se desarrolla en las IES, debe estar interrelacionada con la ciencia, la tecnología y la innovación, siendo esta unión, la base para la transformación y evolución de este tipo de instituciones. También debe tomarse en cuenta que a partir de la esencia de esta función, gestionar el conocimiento está directamente relacionado con el 
proceso administrativo de crear, planear, almacenar y evaluar conocimiento con el fin de compartirla entre las personas y grupos de las instituciones y de organizaciones externas con intereses y necesidades comunes, con el fin de realizar aportes y soluciones a problemáticas existentes y a la innovación.

Atendiendo a lo anteriormente expresado, todo indica que el eje central del proceso es el intelecto de las personas, el mismo que debe ponerse a disposición de los intereses y necesidades del colectivo; atender a las exigencias y desafíos del entorno social y global; al mismo tiempo como una forma de sobresalir en este sector tan complejo y competitivo como lo es la educación superior. Sin embargo, es necesario mencionar que llevar a la práctica estos procesos no ha sido tarea fácil para este tipo de instituciones, especialmente porque se presentan algunas dificultades de tipo organizacional y tecnológico, que limitan el logro de resultados significativos en este (Escorcia y Barros, 2020).

Por lo anterior, la gestión del conocimiento adquiere importancia en las IES en la medida que contribuye a mejorar la gestión interna, promueve la innovación mediante la transferencia de conocimiento, fortalece la cultura investigativa entre profesores y estudiantes, promueve la actualización continua y el desarrollo de habilidades y competencias, e influye significativamente en la toma de decisiones por parte del equipo directivo, permitiendo alcanzar un nivel superior de calidad que genere mayor valor organizacional (Escorcia y Barros.,2020).

\section{Dimensiones de la Gestión del Conocimiento}

Basados en las definiciones anteriores, para efectos de desarrollar esta investigación, se han seleccionado las dimensiones que permitirán el desarrollo de una gestión del conocimiento idóneo y sugerido, a efectos de adaptar y reestructurar los procesos que se llevan a cabo en la Carrera de Educación Primaria en la Universidad Nacional del Santa, en Perú.

Al respecto, Bom y Bolívar, (2015), indican que en cualquier organización que pretenda comenzar un proceso de mejoramiento, es necesario que exista un área donde se lleven adelante las siguientes funciones: a) generación y administración del conocimiento necesario para el desarrollo del sistema (explícito y tácito),

b) gestión de la circulación y transferencia del conocimiento por medio del aprendizaje organizacional y las tecnologías de información y comunicación;

c) registro y almacenamiento del conocimiento organizacional que se produce en los procesos de trabajo durante el cambio y,

(d) utilización del conocimiento como recurso para mejorar el desempeño. Partiendo de lo anterior, para ésta investigación se consideraron como dimensiones, las siguientes: (Ver Cuadro 2) 
Gestión del conocimiento en la carrera de educación primaria en la Universidad Nacional del Santa, Perú

Cuadro 2. Dimensiones de la Gestión del Conocimiento

\begin{tabular}{|c|c|}
\hline \multicolumn{2}{|c|}{ Dimensiones de la Gestión del Conocimiento } \\
\hline 1. Creación det Conociniento. & 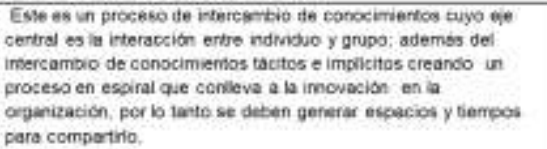 \\
\hline $\begin{array}{l}\text { 2. Transferencis y Amacenamiento del } \\
\text { Conocimiento }\end{array}$ & 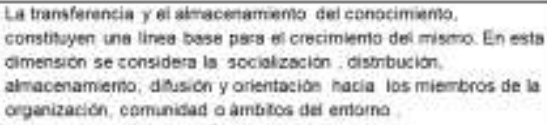 \\
\hline 3. Agilcación y usó dal Cenocimiento & 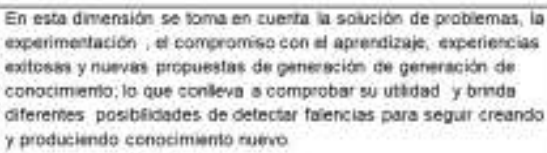 \\
\hline
\end{tabular}

Fuente: Elaboración propia (2020), basado en Tarí y García (2009).

Concebir un proceso de gestión del conocimiento conlleva a desarrollar métodos procedimientos y el uso de herramientas, con el fin de: crear, transferir, almacenar, aplicar y usar el conocimiento. Pero siempre teniendo claro lo que indica Alvarado (2016), quien señala que el conocimiento tiene su centro de acción en los individuos y en general, en las personas que toman decisiones en las organizaciones (capital intelectual).

Este centrar la atención en el capital intelectual, según Escocia y Barros (2020), permite el logro del éxito de las empresas; además se debe tener en cuenta que es el personal 0 recurso humano de cualquier organización, quienes contribuyen a la mejora de los procesos, procedimientos, y al desarrollo de innovaciones; logrado todo por medio de la adaptación y uso de las tecnologías que los apoyan: brindando, claridad, flexibilidad y eficiencia. Así mismo una gestión del conocimiento desarrollada de forma idónea, va más allá del cumplimiento de fases o etapas en un proceso administrativo, dado que constituye el pilar fundamental para el logro de resultados óptimos en las empresas, pero en todo tipo de instituciones de educación superior (IES).

Para obtener los resultados esperados de este tipo de gestión, las instituciones de educación superior (IES), deben disponer del personal, y líderes, en este caso serían las autoridades y directores de cada facultad, escuela o departamento, los que estén a la cabeza, guíen u orienten y faciliten estos procesos. Uno de los factores clave de éxito en la gestión del conocimiento, es crear una cultura orientada al aprendizaje, que se adapte al modelo que la organización desee implementar. Ahora bien, considerando que el conocimiento es un activo intangible estratégico, por tanto, debe ser gestionado por la alta dirección.

Por ello, el respaldo del personal de la alta dirección y los líderes de la organización, es vital para informar y establecer el compromiso de todos los integrantes de la institución para con la gestión del conocimiento; siendo clave crear una cultura que promueva el aprendizaje organizacional, con miras a obtener la organización que se desea (Escorcia y Baros 2020). Lograr los objetivos institucionales con respecto a una gestión del conocimiento idónea, está directamente relacionada con el respaldo financiero que se requiere en esta labor. 
Esencial es además, la motivación constante y los incentivos que la organización ofrece a sus miembros para crear conocimiento; estos juegan un papel importante para crear 0 generar, compartir, usar y aplicar de forma habitual el conocimiento. Con lo antes expresado coinciden, López, Falconi, López y Pomaquero (2018), ellos advierten que todos los esfuerzos e iniciativas para generar conocimiento, deben ser incentivados o estimulados y recompensados, de forma acorde con los aportes que se realicen; bajo un liderazgo que conlleve a una gestión exitosa del conocimiento.

En ese orden de ideas, por medio de la implementación de las dimensiones antes descritas, una gestión del conocimiento dirigida a la obtención de resultados y logro de objetivos, debe ser organizada y orientada a mejorar todos los procesos que implican el manejo del conocimiento y la información en todas las áreas institucionales.

\section{Gestión del Conocimiento en la Carrera de Educación Primaria.}

La escuela de Educación Primaria de la Universidad Nacional del Santa, forma Licenciados en Educación Primaria, capacitados y preparados científica, tecnológica y humanísticamente, para orientar la formación integral de la personalidad del niño y el desarrollo armónico de sus potencialidades intelectuales, afectivas y psicomotoras, de conformidad con su nivel evolutivo. Facilitando la adquisición de conocimientos básicos y desarrollo de destrezas fundamentales que vinculen al educando con las actividades prácticas. Al egresar el docente, en el ejercicio de su profesión, coordina e interactúa con los padres de familia y las organizaciones sociales y culturales de la comunidad; asimismo con otros profesionales de otras áreas del conocimiento (Universidad Nacional del Santa, 2020).

De acuerdo a la descripción anterior sobre la naturaleza de la Carrera de Educación Primaria de la Universidad Nacional del Santa, en Perú; se puede verificar que existen procesos internos de gestión del conocimiento que se llevan a cabo; en ese sentido la gestión del conocimiento se realiza según los recursos existentes, percibiéndose la implementación de métodos, procedimientos, estrategias y demás mecanismos, para desarrollar la función académico-formativa en la carrera, con el fin de la profesionalización de los futuros docentes en educación primaria.

En este caso, la universidad y específicamente la Escuela de Educación, como centro de producción de conocimientos por excelencia, aparte de asegurar el logro de los objetivos, acatando la orientación y normativa institucional, la gerencia debe posibilitar y asegurar al mismo tiempo, los objetivos para el desarrollo de una gestión del conocimiento; como son la identificación de elementos claves, descripción y caracterización del proceso. Tal como lo señalan Bom y Bolívar (2015), algunas estrategias que pueden ser implementadas en la gestión para la trasmisión del conocimiento, pueden ser por ejemplo: la conformación de equipos de investigación en redes nacionales e internacionales, por áreas temáticas afines, producción de artículos de investigación y los libros coeditados, direcciones y codirecciones de tesis de grado, publicaciones en revistas científicas, entre otras.

Escorcia y Barros (2020), se refieren a la gestión del conocimiento que se realiza por medio del proceso formativo, de la siguiente manera: Desde las funciones sustantivas de docencia, las IES están generando y transmitiendo conocimiento a sus estudiantes constantemente, siendo necesario gestionarlo de manera adecuada, para obtener resultados significativos tanto en su rendimiento, como para el desarrollo institucional. Por otro lado, en el 
Gestión del conocimiento en la carrera de educación primaria en la Universidad Nacional del Santa, Perú

quehacer académico los estudiantes desarrollan proyectos, trabajos investigativos, ensayos y otros documentos, que pueden ser aprovechados por otros miembros de la institución y/o actores del sector externo, dándole aplicabilidad real a esa información.

\section{Metodología}

Para lograr el objetivo de la investigación en cuanto a la herramienta para mejorar la gestión del conocimiento en la carrera de educación primaria de la Universidad Nacional del Santa, en Perú, se realizó un tipo de investigación descriptiva, con diseño de campo; que se centra en describir situaciones, eventos o hechos, recolectando datos sobre una serie de cuestiones, efectuando mediciones y explicando lo que sucede en un momento dado (Cabezas, Andrade y Torres, 2018).

La población muestral, estuvo constituida por la totalidad de estudiantes de la carrera de Educación Primaria de la Universidad Nacional del Santa, en Perú, Correspondiente a 120 estudiantes, según el registro de alumnos inscritos facilitado por secretaria docente de dicha facultad. El criterio para determinar la población muestral fue: la opinión directa del grupo de estudiantes; se obtuvo la percepción de cada uno, por medio de la técnica de recolección de información o encuesta. Lo que permitió diagnosticar la situación sobre la gestión del conocimiento, que se desarrolla en la Escuela de Educación Primaria de la Universidad Nacional del Santa (UNS), en Perú.

Como instrumento: se empleó el cuestionario, constituido por los siguientes indicadores, tomando como referencia lo planteado por Tarí y García (2009), sobre las dimensiones que definen la gestión del conocimiento: 1. Creación del conocimiento; 2. Transferencia y almacenamiento del conocimiento; 3. Aplicación y uso del conocimiento; 4. Mecanismos para crear, transferir, difundir y aplicar conocimientos; 5 . Modelo para gestionar el conocimiento.

Esta información estuvo distribuida en un cuestionario de 07 ítems, validado con juicio de expertos; y con una confiabilidad obtenida después de aplicar la medida estadística Alfa de Cronbach con un valor de 0,896/ Bueno. Cada ítem fue estructurado para las respuestas con la escala de Likert y las siguientes valoraciones: Totalmente en desacuerdo (1), En desacuerdo (2), Neutral (3), De acuerdo (4) y Totalmente de acuerdo (5). Se utilizó la estadística descriptiva con cuadros y gráficos Estadísticos.

\section{Resultados}

Por medio del diagnóstico surgido de la indagación a la población conformada por 120 estudiantes, se determina que es necesario desarrollar una gestión del conocimiento, acorde a las necesidades y percepciones de los encuestados. Tal como se observa en las siguientes tablas relacionadas con las preguntas sobre gestión del conocimiento que se realiza en la escuela de educación, en la carrera de educación primaria de la UNS en Perú, los resultados fueron los siguientes:

Tabla 1. Gestión del conocimiento en la carrera de Educación Primaria de la UNS

Fuente: Elaboración propia (2020).

\begin{tabular}{lccc}
\hline & & $\mathbf{N}^{\circ}$ & $\%$ \\
\hline Deficiente & {$[30-69]$} & 3 & 2.5 \\
Regular & {$[70-110]$} & 73 & 60.8 \\
Bueno & {$[111-150]$} & 44 & 36.7 \\
\hline & TOTAL & 120 & 100,0 \\
\hline
\end{tabular}


Gráfico 1. Gestión del conocimiento en la carrera de Educación Primaria de la UNS

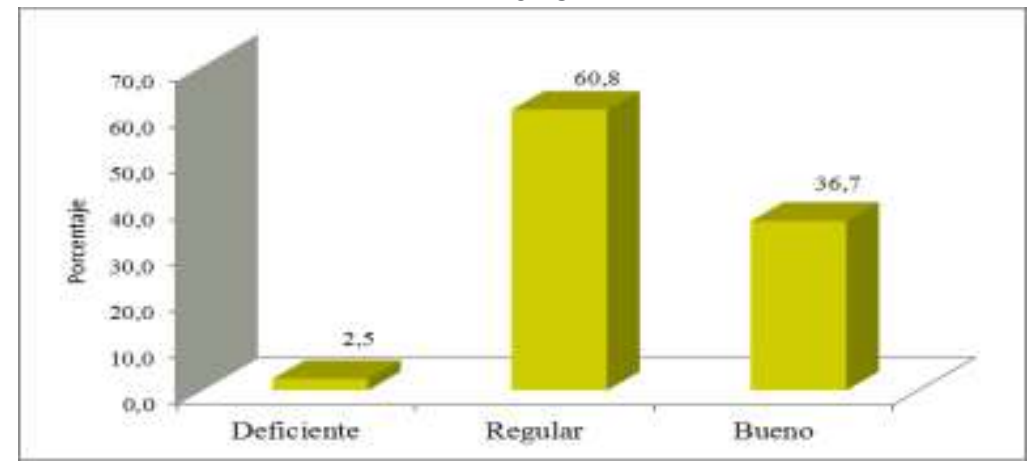

Fuente: Elaboración propia (2020).

De esta forma la tabla y gráfico 1, presentan la valoración por parte de los 120 estudiantes de la carrera de educación primaria, sobre la gestión del conocimiento en la escuela de educación primaria de la UNS. En ese sentido el 2,5\% de los estudiantes encuestados han expresado que la gestión del conocimiento está en un nivel deficiente; el $60,8 \%$ de los estudiantes indicó que está en un nivel regular y el 36,7\% indicó que la gestión del conocimiento está en un nivel bueno. Estos resultados nos indican que es necesario mejorar el proceso de gestión del conocimiento en esta escuela de educación, Rodríguez (2006); ya que por medio de la percepción de los estudiantes, se califica como regular la gestión que se lleva a cabo en esta escuela.

Tabla 2. Percepciones: Creación de conocimiento que se realiza en la Carrera de Educación Primaria UNS

\begin{tabular}{|c|c|c|c|}
\hline & & $\mathrm{N}^{\circ}$ & $\%$ \\
\hline Deficiente & [18-41] & 3 & 2.5 \\
\hline Regular & [42-66] & 74 & 61.7 \\
\hline Bueno & [67-90] & 43 & 35.8 \\
\hline \multicolumn{2}{|c|}{ TOTAL } & 120 & 100,0 \\
\hline
\end{tabular}

Fuente: Elaboración propia (2020). 
Gestión del conocimiento en la carrera de educación primaria en la Universidad Nacional del Santa, Perú

Gráfico 2. Percepciones sobre creación de conocimiento en la Carrera de Educación Primaria UNS.

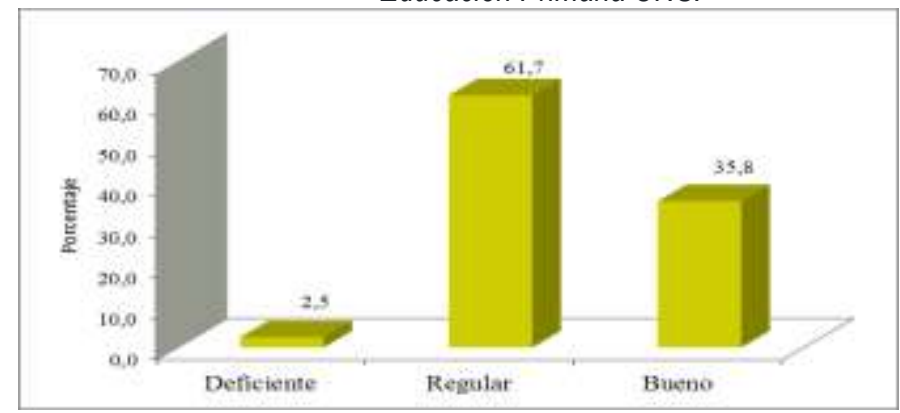

Fuente: Elaboración propia (2020).

La tabla y gráfico 2, resume la opinión de los 120 estudiantes sobre la creación de conocimiento en la carrera de educación primaria, calificando ésta en el nivel bueno el 35,8\% de los estudiantes; el 2,5\% de los estudiantes lo ubica en un nivel deficiente, y el puntaje mayor $61,7 \%$ opina que la creación del conocimiento está en un nivel regular. Con los resultados se evidencia la ausencia de políticas para establecer mecanismos claros y dinámicos para la creación de conocimiento, Tobón y Rojas (2006). Se sugiere la apertura al cambio por parte de estudiantes, docentes y autoridades, siendo esta una característica con la que debe contar una organización que aprende, además desarrollar la creatividad en las actividades que se realizan, y en las formas y procedimientos para generar conocimiento.

Tabla 3. Percepciones sobre el almacenamiento y transferencia del conocimiento en la Carrera de Educación Primaria UNS

\begin{tabular}{llcc}
\hline & & $\mathbf{N}^{\circ}$ & $\%$ \\
\hline Deficiente & {$[18-41]$} & 5 & 4.2 \\
Regular & {$[42-66]$} & 78 & 65.0 \\
Bueno & {$[67-90]$} & 37 & 30.8 \\
\hline & TOTAL & 120 & 100,0 \\
\hline
\end{tabular}

Fuente: Elaboración propia (2020). 
Gráfico 3. Percepciones sobre el almacenamiento y transferencia del conocimiento en la Carrera de Educación Primaria UNS

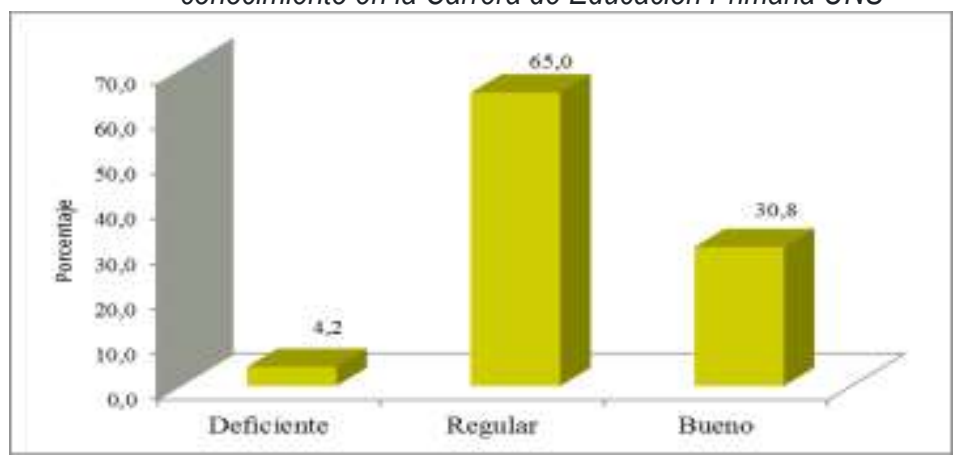

Fuente: Elaboración propia (2020).

En la tabla y gráfico 3, se presenta la reacción de los estudiantes encuestados en relación a los mecanismos para almacenar y transferir el conocimiento en la carrera de educación primaria, en ese sentido el $4,2 \%$ de los estudiantes responden que los mecanismos para almacenar y transferir el conocimiento están en un nivel deficiente, otro grupo de estudiantes con mayor frecuencia $(65,0 \%)$ mencionan que se encuentra en un nivel regular y el $30,8 \%$ de los encuestados dijeron que es bueno.

Por lo anterior, se puede afirmar, que el almacenamiento y transferencia del conocimiento en la carrera, se realiza de manera regular; la universidad actualmente cuenta con un repositorio institucional, en el cual desde el año 2015 se viene construyendo una base de datos con los resultados de las investigaciones científicas de docentes y estudiantes, por lo que se puede inferir que no es suficiente, debido a que se necesita construir más bases de datos para almacenar todo tipo de productos de investigación y creación de conocimiento; como por ejemplo los productos de las propuestas de innovación, sistematización de experiencias educativas, producción de textos diversos, edición de textos, etc.

De esta manera, la gestión del conocimiento se está concibiendo como objetivo para lograr la mejora de los resultados de una organización, Tarí y García (2009). En ésta dimensión se sugiere la socialización de los nuevos conocimientos generados, a través de la distribución en nuevas bases de datos; y se interactúe con la comunidad a partir de la transferencia y difusión.

Tabla 4. Percepciones sobre la aplicación y uso del conocimiento en la Carrera de Educación Primaria UNS

\begin{tabular}{lccc}
\hline & & $\mathbf{N}^{\circ}$ & $\%$ \\
\hline Deficiente & {$[05-11]$} & 5 & 4.2 \\
Regular & {$[12-18]$} & 40 & 33.3 \\
Bueno & {$[19-25]$} & 75 & 62.5 \\
\hline \multicolumn{2}{c}{ TOTAL } & 120 & 100,0 \\
\hline
\end{tabular}

Fuente: Elaboración propia (2020). 
Gestión del conocimiento en la carrera de educación primaria en la Universidad Nacional del Santa, Perú

Gráfico 4. Percepciones sobre la aplicación y uso del conocimiento en la Carrera de Educación Primaria UNS

Fuente: Elaboración propia (2020).

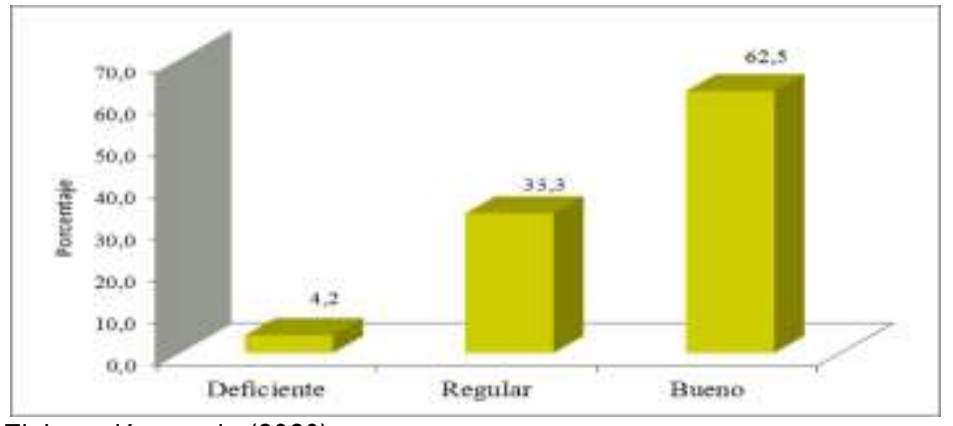

La tabla y gráfico 4 muestra la información sobre la aplicación y uso del conocimiento en la carrera de educación primaria de la UNS, considerando con mayor puntaje, un $62,5 \%$, que se ubica en un nivel bueno, otro grupo de estudiantes el $33,3 \%$ considera que está en un nivel regular, y por último otro grupo de estudiantes del $4,2 \%$ califica que es de un nivel deficiente. Al analizar esta dimensión, se verifica que para desarrollar una buena gestión, en cuanto a la generación de conocimiento para la solución problemas actuales existentes, a la experimentación en situaciones académicas con nuevos conocimientos, y a la práctica de experiencias exitosas e iniciativas para generar nuevos conocimientos, se necesita del compromiso de estudiantes y docentes por adquirir el aprendizaje.

Se infiere a partir de los resultados que los estudiantes creen importante la aplicación del conocimiento creado en la propia universidad, por lo tanto se debe considerar los resultados de este estudio, y la herramienta proporcionada, como un proceso más completo, de gran utilidad (Mesa, 2006).

Tabla 5. ¿Considera usted importante que la carrera cuente con mecanismos para crear, transferir, difundir y aplicar conocimientos?

\begin{tabular}{lcc}
\hline & $\mathbf{N}^{\circ}$ & $\%$ \\
\hline Totalmente en & 4 & 3,3 \\
desacuerdo & 1 & 0.8 \\
En desacuerdo & 5 & 4,2 \\
Neutral & 36 & 30,0 \\
De acuerdo & 74 & 61.7 \\
Totalmente de acuerdo & 120 & 100,0 \\
\hline \multicolumn{1}{r}{ TOTAL } &
\end{tabular}

Fuente: Elaboración propia (2020). 
Gráfico 5. ¿Considera usted importante que la carrera cuente con mecanismos para crear, transferir, difundir y aplicar conocimientos?

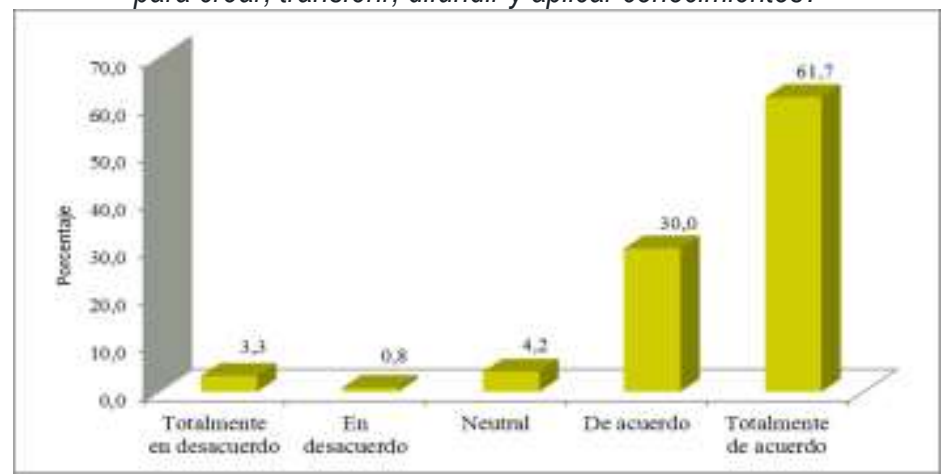

Fuente: Elaboración propia (2020).

En la tabla y gráfico 5, sobre la importancia de que la carrera cuente con mecanismos para crear, transferir, difundir y aplicar conocimientos, la mayor frecuencia fue de un $61,7 \%$, donde los estudiantes están totalmente de acuerdo con lo planteado; otro grupo de estudiantes el 30,0\% están de acuerdo. Como es visible el $91,7 \%$ de los estudiantes están de acuerdo y totalmente de acuerdo con el considerando mencionado, y en desacuerdo solo estuvo el $0,8 \%$. La herramienta que en este estudio se sugiere para gestionar el conocimiento, responderá a las expectativas de los estudiantes, pero también responde a los estándares 12, 22, 23, 24, que hacen referencia a la producción científica e innovación que se debe desarrollar en el programa de estudios de Educación Primaria (Sineace, 2016).

Tabla 6. ¿Considera usted en la carrera no se crea, no se transfiere, no se difunde y por ende no se aplica conocimiento?

\begin{tabular}{lcc}
\hline & $\mathbf{N}^{\circ}$ & $\%$ \\
\hline Totalmente en desacuerdo & 27 & 22,5 \\
En desacuerdo & 35 & 29.2 \\
Neutral & 36 & 30,0 \\
De acuerdo & 14 & 11,7 \\
Totalmente de acuerdo & 8 & 6.7 \\
\hline \multicolumn{1}{r}{ TOTAL } & 120 & 100,0 \\
\hline
\end{tabular}

Fuente: Elaboración propia (2020). 
Gestión del conocimiento en la carrera de educación primaria en la Universidad Nacional del Santa, Perú

Gráfico 6. ¿Considera usted en la carrera no se crea, no se transfiere, no se difunde y por ende no se aplica conocimiento?

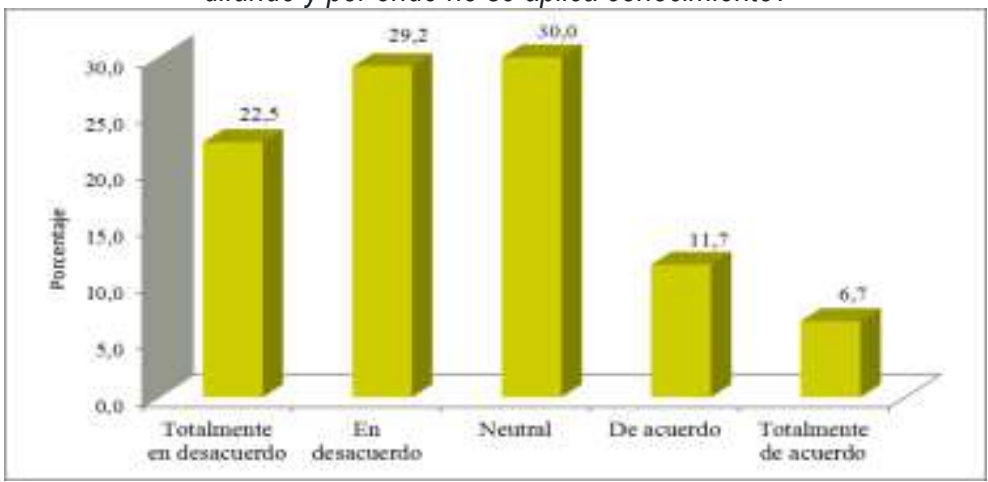

Fuente: Elaboración propia (2020).

La Tabla y gráfico 6 muestra la percepción de los 120 estudiantes en cuanto a las consideraciones referidas a la no creación, no transferencia, no difusión y por ende no se aplica conocimiento en la carrera de educación primaria de la UNS; donde, se obtuvo que el $22,5 \%$ de los estudiantes están totalmente en desacuerdo con la referencia mencionada, el $29,2 \%$ están en desacuerdo, el 30,0\% se mantienen neutral, el 11,7\% están de acuerdo y el $6,7 \%$ están totalmente de acuerdo. Como es visible el $51,7 \%$ de los estudiantes están totalmente en desacuerdo y en desacuerdo con el considerando mencionado, lo que implica que se requiere de estrategias y una gestión de conocimiento donde las dimensiones de este proceso se lleven a cabo con eficiencia.

Tabla 7. ¿Cree usted que la carrera debe contar con un modelo para gestionar el conocimiento?

\begin{tabular}{lll}
\hline & $\mathrm{N}^{\circ}$ & $\%$ \\
\hline -Totalmente en desacuerdo & 3 & 2,5 \\
-En desacuerdo & 0 & 0.0 \\
-Neutral & 5 & 4,2 \\
-De acuerdo & 49 & 40,8 \\
-Totalmente de acuerdo & 63 & 52.5 \\
\hline \multicolumn{1}{c}{ TOTAL } & 120 & 100,0 \\
\hline
\end{tabular}

Fuente: Elaboración propia (2020) 
Gráfico 7. ¿Cree usted que la carrera debe contar con un modelo para gestionar el conocimiento?

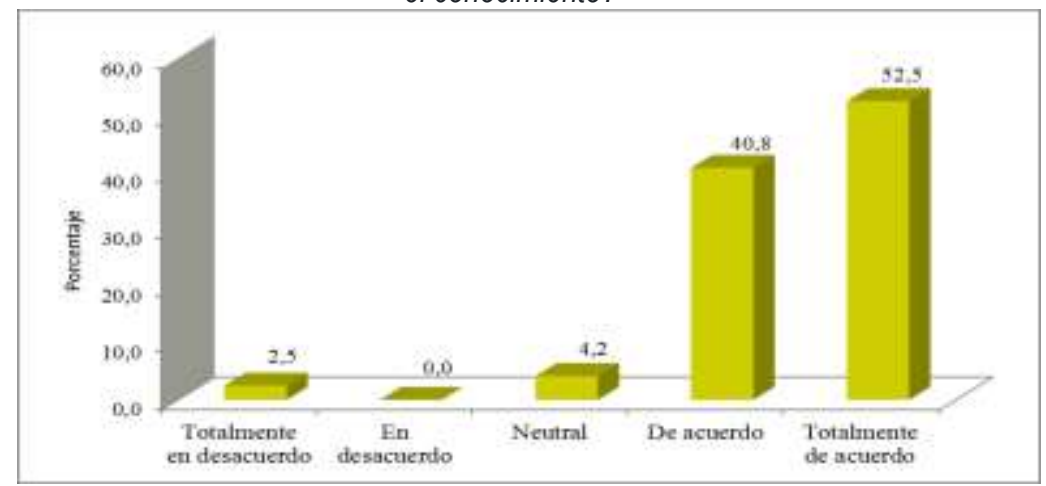

Fuente: Elaboración propia. (2020)

La Tabla y gráfico 7 presenta la opinión de los 120 estudiantes, sobre el hecho que, la carrera debe contar con un modelo para gestionar el conocimiento, al respecto se obtuvo que el 40,8\% está de acuerdo, el resto, un 4,2\% se mostró neutral y un 2,5\% estuvo totalmente en desacuerdo en su reacción hacia este planteamiento. En cuanto a estos resultados, se percibe como necesaria una gestión del conocimiento acorde con las expectativas de los estudiantes, y la literatura existente sobre este tema.

\section{Herramienta de Mejora Continua para la Gestión del Conocimiento de la Escuela de Educación Primaria}

En función de los resultados de esta investigación, se puede verificar la problemática existente, en varios de los indicadores tales como la gestión y creación del conocimiento, así como los mecanismos para almacenar y transferir el conocimiento en la escuela de educación primaria de la UNS, tales como siendo propicio elaborar y sugerir una herramienta para ser aplicada y luego evaluar su efectividad en la gestión del conocimiento. Ésta debe asumirse como un proceso dinámico, que posea como aspectos básicos a desarrollar, la creación, almacenamiento, transferencia, aplicación y uso del conocimiento, como base de la nueva gestión a emprender. Tal como lo señalan, Bom y Bolívar (2015), la gestión del conocimiento se centra en desarrollar el conocimiento en las organizaciones reconociendo sus fases de adquisición, almacenamiento, transformación, distribución y la utilización, con la finalidad de lograr ventajas competitivas y comparativas. Asimismo, se verifica la problemática existente.

La mejora continua, según Tórrez (2015), es consecuencia de una forma ordenada de administrar y mejorar los procesos, identificando causas o restricciones, estableciendo nuevas ideas y proyectos de mejora, llevando a cabo planes, estudiando y aprendiendo de los resultados obtenidos y estandarizando los efectos positivos para proyectar y controlar el nuevo nivel de desempeño, Por lo tanto, su aplicación optimizará los procesos de manejo de la información y el conocimiento que se generen, tanto en lo interno (institución, personal, procesos), como para la resolución de problemáticas existentes en el entorno comunitario y social 
Gestión del conocimiento en la carrera de educación primaria en la Universidad Nacional del Santa, Perú

Ante todo, es importante considerar que para lograr la mejora continua se requiere la participación de todos los miembros de la institución y de los departamentos que estén directamente relacionados con la gestión del conocimiento: autoridades, docentes, estudiantes, administrativos, miembros de la sociedad y grupos de interés, que deseen participar e integrarse en equipos de trabajo para llevar a cabo las tareas dispuestas para este fin.

Este grupo de personas debe poseer, competencias y conductas, personales y profesionales que favorezcan el compromiso y apoyo al proceso que se desea impulsar; al tiempo que debe estar encabezado por los líderes de la institución. De acuerdo a esto, Rujano, Jacobo, Núñez y Anaya (2020), destacan la importancia del involucramiento del personal que realiza funciones estratégicas, a quienes se les integra en los equipos de trabajo, se les capacita y forma, para que desarrollen un buen liderazgo y eleven su desempeño, alcanzando la satisfacción personal y profesional.

La herramienta de mejora continua para la gestión del conocimiento, de la Escuela de Educación Primaria (EEP), de la Universidad Nacional del Santa (UNS), en Perú; que se estima pueda desarrollarse a futuro, amerita un liderazgo efectivo, en el sentido que deben realizarse tareas y procesos, que satisfagan las necesidades y expectativas surgidas de los resultados arrojados por el cuestionario aplicado a los estudiantes de la mencionada escuela. Por lo cual, la gestión debe estar liderada por un profesional en el área, capacitado e idóneo para desempeñar esta función. Este aspecto lo dejan en claro, Pozo, Ruíz, Vigo y Flores, (2020), cuando señalan que: la función gerencial, basada en el conocimiento, con orientaciones hacia el dominio gerencial, hace énfasis sobre la preparación y la práctica administrativa que se adquiere, pero debe prepararse también en función de enseñar a ser, enseñar a aprender y que aprenda al enseñar.

También indican Pozo, et al., (2020), que para ser un gerente del talento humano, debe tener la capacidad de aprender, y que esté socialmente comprometido con la comunidad a su vez, que se mantenga en proceso permanente de formación y crecimiento profesional.

En correspondencia con lo anterior, la herramienta de gestión del conocimiento a implementar será, el ciclo Deming o ciclo PHVA: "Planificar - Hacer - Verificar - Actuar", que está ligado a la planificación, implementación y control, para los procesos de un sistema de gestión, Araujo, et al., (2020). Esta herramienta de mejora inducirá el proceso a través de 4 pasos que son: planear, hacer, verificar y actuar; configurada en una secuencia lógica y concatenada para la obtención programada de resultados en cuanto a las dimensiones seleccionadas para implementar la gestión del conocimiento: creación, de almacenamiento, de transferencia, de aplicación y uso del conocimiento para el logro de fines y objetivos planteados.

El ciclo de Deming o ciclo PHVA, que se relaciona con la planificación, implementación, control y mejora continua, se empleará como herramienta para desarrollar la gestión del conocimiento en la E.E.P de la UNS. Basada en una realización constante (cíclica) de etapas específicas, como parte de la administración estratégica que permitirá: analizar: la misión, visión, objetivos, entorno (interno y externo); decidir: políticas y mecanismos para la obtención de ventajas competitivas; proponer acciones: emprender estrategias adecuadas y pertinentes para gestionar el conocimiento.

En torno a lo anterior, el creador del ciclo PHVA, Deming indica que a través de un proceso de mejoramiento continuo de la calidad, es posible que una organización pueda tener éxito y 
sobrevivir en el mercado. Es por ello, que las organizaciones, deben establecer mecanismos para medirlas y así proyectar su potencial y correcto desarrollo (Pozo, Ruíz, Vigo y Flores; 2020).

\section{Figura 1. Herramienta de mejora continua - Ciclo de Deming}

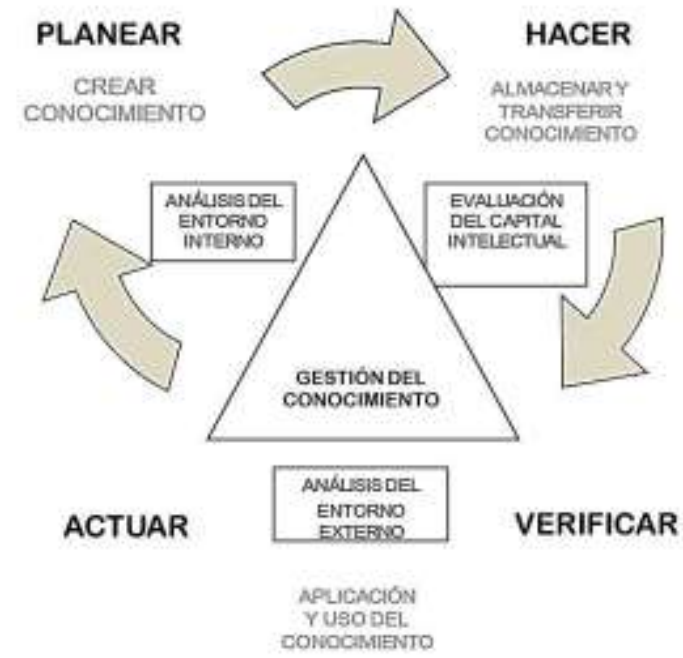

Fuente: Elaboración propia (2020). En base a los fundamentos Deming (1989) citado por Pérez (2017)

El ritmo del cambio o avance del ciclo, dependerá del número de acciones de mejoramiento que se efectúen y de la efectividad con que éstas se realicen; por ello, es importante que este enfoque sea una idea internalizada por completo en la conducta de todos los miembros de la institución, para convertirse en una filosofía de trabajo y de vida. Sin embargo, para ciertos procesos muy utilizados u obsoletos, quizá el mejoramiento continuo no sea el remedio idóneo, siendo oportuno realizar una revisión teórica y encontrar la herramienta adecuada según sea el caso (Araujo, Orellana, Cortés, Gonzalo y Zambrano, 2020)

Para finalizar se debe tener en cuenta, que la herramienta que aquí se presenta, será a efectos de orientar y ejemplificar como desarrollar un proceso de gestión del conocimiento, para el caso de investigación. Para la estructuración o construcción teórico-práctica, se deben considerar todos los aspectos descritos y explicarlos detalladamente (con la organización de contenidos, pasos, etapas, etc.), lo que conlleva a hacer esta investigación en dos etapas, sirviendo además como una guía para implantar la gestión del conocimiento de forma estratégica, en la escuela de educación primaria, de la Universidad Nacional del Santa (UNS). Conclusiones

La gestión del conocimiento como proceso administrativo conlleva a una secuencia de pasos que siendo desarrollados de manera adecuada y eficiente, contribuye a la obtención de resultados significativos; tanto para la eficiencia de la función educativa, en el caso de las 
Gestión del conocimiento en la carrera de educación primaria en la Universidad Nacional del Santa, Perú

instituciones de educación superior, como para la solución de problemáticas existentes hacia lo interno y externo de cualquier tipo de empresa.

Las opiniones y percepciones recogidas con esta investigación, de parte de los estudiantes sobre la gestión del conocimiento que se lleva a cabo en la Escuela de Educación Primaria de la Universidad Nacional del Santa en Perú, indica que amerita cambios y el fortalecimiento de los procesos y procedimientos.

De este proceso de indagación se infiere que la gestión se ejecuta con deficiencias en algunos de los procesos, tales como la gestión y creación del conocimiento, además de los mecanismos para almacenar y transferir el conocimiento, lo que los aleja del deber ser de éste proceso de enseñanza y aprendizaje.

Ante esta situación se indica implementar la herramienta de mejora continua, sugerida para este fin (Ciclo PHVA) o ciclo de Deming, estrategia con la que podrán resolverse las dificultades presentes para desarrollar una buena gestión del conocimiento, que lleve al logro de objetivos planteados y resultados esperados.

Los miembros de la institución forman parte esencial de cualquier proceso de gestión; la herramienta de mejora continua o ciclo PHVA requiere a la inclusión de personal idóneo y competente y comprometido con los valores, políticas y fines de la institución, por lo que se debe promover la formación y capacitación continua, como estrategia de motivación, asimismo definir criterios para incentivas las buenas prácticas que exige este proceso.

La creación o generación, transmisión, divulgación, uso y aplicación del conocimiento indicado como fases del ciclo, depende de la iniciativa y trabajo de todo el personal de la institución encargados de esta área. En tal sentido, la participación de las autoridades del programa de educación, es vital para comunicar entre el resto del personal, docente, administrativo, obrero, estudiantes y comunidad educativa en general, el compromiso que deben adquirir con la gestión del conocimiento y con el aprendizaje organizacional; sobre todo es imprescindible prever la importancia del respaldo financiero por parte de la institución, para llevar a cabo estas acciones.

Se resalta la importancia en que la gestión del conocimiento debe desarrollarse realizando una mayor inversión económica, de recursos materiales y tecnológicos, con la amplitud de los procesos en espacio y tiempo, la participación activa del personal requerido para este fin; la inclusión de los estudiantes ya que aportan gran parte del conocimiento e información que se genera. El uso y aplicación efectiva del conocimiento, será la clave para la solución de problemáticas existentes en la universidad y el entorno; además de servir de insumo para la innovación educativa y tecnológica.

De acuerdo a lo anterior, la implementación de esta herramienta para la gestión del conocimiento debe estar basada en el planteo de objetivos institucionales bien definidos, de no haber una participación consensuada, la gestión no tendrá éxito; lo cual como ya se mencionó, depende de la disposición al cambio del conjunto de personas que hacen vida en la institución; la obtención de resultados favorables también dependerá del esfuerzo que se le imprima; siendo esta herramienta muy útil ya que permite revisar cada una de las etapas para determinar donde se están presentando dificultades y resolverlas. 


\section{Referencias Bibliográficas}

Alvarado, Lisandro (2016). Modelo de gestión de calidad para los centros de investigación universitarios venezolanos. Tesis de Grado para optar al: doctorado en Ciencias sociales. Universidad del Zulia (LUZ). Venezuela.

Araujo, Rubén; Orellana, Marcos; Cortés, Gonzalo., y Zambrano, Juan (2020). Principios de Gestión de la Calidad en Estudios a Distancia de Universidades Privadas. Revista Venezolana de Gerencia (RVG). Año 25 Número especial 3, Venezuela. (Pp.460481). http://dx.doi.org/10.37960/rvg.v25i3.33383.

Barbón, Olga., y Fernández, Jorge (2017). Rol de la gestión educativa estratégica, en la gestión del conocimiento, la ciencia, la tecnología y la Innovación, en la educación Superior. Revista Educación Médica. Elsevier, Volume 19, Issue 1. España. (Pp.5155). https://doi.org/10.1016/j.edumed.2016.12.001

Bom, Yomeida y Bolívar, Juan (2015). Gestión del conocimiento y activos intangibles en universidades públicas: Perspectiva de análisis. Revista Venezolana de Gerencia (RVG). Año. 23, No. 82, 2018, Venezuela.(Pp.457- 478). http://dx.doi.org/10.37960/revista.v23i82.23761.

Briceño, Margarita; Correa, Susana; Valdés, Michel y Hadweh, Marcelo (2020). Modelo de Gestión Educativa para Programas en Modalidad Virtual de Aprendizaje. Revista de Ciencias Sociales. Vol, XXVI, $\mathrm{N}^{\circ}$ 1. Venezuela. (Pp.286-298). http://dx.doi.org/10.31876/rcs.v26i2.32442.

Cabezas, Edison; Andrade, Diego y Torres, Johana (2018). Introducción a la Metodología e la Investigación Científica. Universidad de las Fuerzas Armadas -ESPE. Primera edición electrónica. Ecuador. Disponible en: http://www.repositorio.espe.edu.ec/handle/21000/15424.

Escorcia, Jey y Barros, David (2020). Gestión del conocimiento en Instituciones de Educación Superior: Caracterización desde una reflexión teórica. Revista de Ciencias Sociales. Vol, $\quad X X V I, \quad N^{\circ} \quad 3 . \quad$ Venezuela. https://dialnet.unirioja.es/servlet/articulo?codigo $=7565469$

Fernández, Leonardo y Soto, Adenis (2015). Gestión del sistema tecnológico de contenidos en la modalidad de educación a distancia. Revista Opción, Año 31, No. Especial 6 (2015). Venezuela. (Pp.261-283). Disponible en: https://www.redalyc.org/pdf/310/31045571017.pdf.

Godoy, Martha; Mora, Janeth y Liberio, Francisco (2017). Gestión del conocimiento para el desarrollo de organizaciones inteligentes. Revista Publicando. 3(9), Ecuador. (Pp.660-673).

Disponible https://revistapublicando.org/revista/index.php/crv/article/view/393.

Isaac, Rob; Herremans, Irene y Nazari, Jamal (2017). Knowledge management in an innovative virtual company. International Journal of Learning and Intellectual

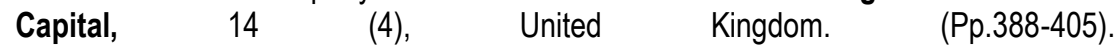
https://doi.org/10.1504/IJLIC.2017.087335.

López, José; López, José; Falconi, Luis y Pomaquero, Juan (2018). Gestión del conocimiento en las organizaciones: fundamentos, metodologías y praxis. Revista Contribuciones a la Economía. Ecuador. Disponible en: https://eumed.net/rev/ce/2018/3/gestionconocimiento.html 
Gestión del conocimiento en la carrera de educación primaria en la Universidad Nacional del Santa, Perú

Mejía, Adriana; Vesga, Alejandro y Gaviria, Margarita (2018). Gestión del conocimiento científico en la Universidad de Antioquia: integración de herramientas para la formulación de una estrategia. Revista Innovar, 28(69), Colombia. (Pp.71-84). http://dx.doi.org/10.15446/innovar.v28n69.71697.

Mesa, Yuniet (2006). De la gestión de información a la gestión del conocimiento. Revista Acimed, 14(1). Cuba. Disponible $\leq h t t p: / s c i e l o . s l d . c u / s c i e l o . p h p ? s c r i p t=s c i \_a r t t e x t \& p i d=\$ 1024-$

94352006000100002\&lng=es\&nrm=iso>.

Pérez, María (2017). Implementación de herramientas de control de calidad en MYPEs de confecciones y aplicación de mejora contínua PHRA. Revista Industrial Data, vol. 20, núm. $\quad 2 . \quad$ Perú. (Pp. 95-100). Disponible en: https://www.redalyc.org/pdf/816/81653909013.pdf

Pérez, Mario. (2016). Gestión del conocimiento: Orígenes y evolución. Revista El profesional de la Información, 25(4), España. (Pp. 526-534). https://doi.org/10.3145/epi.2016.jul.02

Pozo, Carlos; Ruíz, José; Vigo, Elsa y Flores, Florencio (2020). Tendencias gerenciales en las organizaciones: una mirada teórica. Revista Venezolana de Gerencia (RVG). Año 25, $\begin{array}{llll}\mathrm{N}^{\circ} & 91 . & \text { Venezuela. } & \text { (Pp.1095-1113). }\end{array}$ https://produccioncientificaluz.org/index.php/rvg/article/view/33184

Rodríguez, David. (2006). Modelos para la creación y gestión del conocimiento: una aproximación teórica. Revista Educar, 37. España. (Pp.25-39). Disponible en: https://www.researchgate.net/publication/28160293_Modelos_para_la_creacion_y_ges tion_del_conocimiento_una_aproximacion_teorica.

Rujano, Martha; Jacobo, Adelina; Núñez, Octavio y Anaya, Ana. (2020). Mejora continua e innovación en agroempresa mexicana: Modelo Self Lead Team. Revista Venezolana de Gerencia (RVG). Año 25 No. 91. Venezuela. (Pp. 796-810). https://produccioncientificaluz.org/index.php/rvg/article/view/33167

SINEACE (2016) Modelo de Acreditación para Programas de Estudios de Educación Superior Universitaria. Sistema Nacional de Evaluación, Acreditación y Certificación de la Calidad Educativa -Sineace. Perú. Disponible en: https://www.sineace.gob.pe/wp-content/uploads/2014/08/Anexo-1-nuevo-modeloprogramas-Resolucion-175.pdf.

Tarí, Juan., y García, Manuel (2009). Dimensiones de la gestión del conocimiento y de la gestión de la calidad: una revisión de la literatura. Revista Investigaciones Europeas de Dirección y Economía de la Empresa. Vol. 15, № 3. España. (Pp.139.152). https://doi.org/10.1016/S1135-2523(12)60105-1.

Tobón, Sergio, y Núñez, Ariel. (2006). La gestión del conocimiento desde el pensamiento complejo: un compromiso ético con el desarrollo humano. Revista Escuela de Administración de Negocios. (58), Perú. (Pp. 27-40). http://dx.doi.org/10.21158/01208160.n58.2006.35

Tórrez, María (2015). La gestión administrativa y su impacto en la mejora continua Hacia la calidad en la empresa Matagalpa Coffee Group, en el municipio de Matagalpa, departamento de Matagalpa, 2013-2014. Tesis para optar al título de Master en Gerencia Empresaria. Universidad nacional autónoma de Nicaragua. 